\title{
Human embryonic stem cell-derived neurons establish region-specific, long-range projections in the adult brain
}

\author{
Julius A. Steinbeck • Philipp Koch • \\ Amin Derouiche $\cdot$ Oliver Brüstle
}

Received: 5 January 2011/Revised: 28 May 2011/ Accepted: 17 June 2011/Published online: 21 July 2011

(C) The Author(s) 2011. This article is published with open access at Springerlink.com

\begin{abstract}
While the availability of pluripotent stem cells has opened new prospects for generating neural donor cells for nervous system repair, their capability to integrate with adult brain tissue in a structurally relevant way is still largely unresolved. We addressed the potential of human embryonic stem cell-derived long-term self-renewing neuroepithelial stem cells (lt-NES cells) to establish axonal projections after transplantation into the adult rodent brain. Transgenic and species-specific markers were used to trace the innervation pattern established by transplants in the hippocampus and motor cortex. In vitro, lt-NES cells formed a complex axonal network within several weeks after the initiation of differentiation and expressed a composition of surface receptors known to be instrumental in axonal growth and pathfinding. In vivo, these donor cells adopted projection patterns closely mimicking endogenous projections in two different regions
\end{abstract}

Electronic supplementary material The online version of this article (doi:10.1007/s00018-011-0759-6) contains supplementary material, which is available to authorized users.

J. A. Steinbeck · P. Koch · O. Brüstle (凹)

Institute of Reconstructive Neurobiology, Life and Brain Center,

University of Bonn and Hertie Foundation,

53127 Bonn, Germany

e-mail: brustle@uni-bonn.de

J. A. Steinbeck

Department of Neurology, University of Bonn Medical Center, 53127 Bonn, Germany

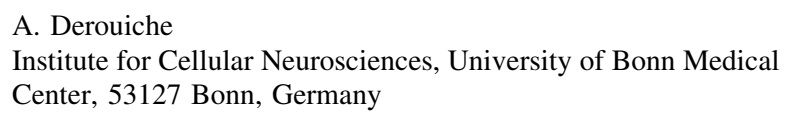

A. Derouiche

Institute of Anatomy II, University of Frankfurt,

60590 Frankfurt, Germany of the adult rodent brain. Hippocampal grafts placed in the dentate gyrus projected to both the ipsilateral and contralateral pyramidal cell layers, while axons of donor neurons placed in the motor cortex extended via the external and internal capsule into the cervical spinal cord and via the corpus callosum into the contralateral cortex. Interestingly, acquisition of these region-specific projection profiles was not correlated with the adoption of a regional phenotype. Upon reaching their destination, human axons established ultrastructural correlates of synaptic connections with host neurons. Together, these data indicate that neurons derived from human pluripotent stem cells are endowed with a remarkable potential to establish orthotopic long-range projections in the adult mammalian brain.

Keywords Neural stem cells - Transplantation · Axon outgrowth $\cdot$ Synaptogenesis

\section{Introduction}

Recent progress with the derivation of neural stem cells from embryonic [1-3], fetal [4-6], and adult [7] sources provides interesting prospects for regenerative medicine [8]. However, the capability of grafted neurons to integrate and in particular to establish appropriate long-range projections in the adult brain has been a matter of controversy. Pioneering studies employing primary fetal human donor cells $[9,10]$ showed a substantial capacity for axonal outgrowth from telencephalic transplantation sites. However, massive in vitro expansion of neural cells was in some studies associated with impaired axonal outgrowth [11-13], while other studies reported extensive or enhanced axonal outgrowth even after extensive pre-transplant in vitro proliferation of the donor cells $[2,14,15]$. Site of implantation, age of the 
transplant recipient, presence and extent of local lesions, and glial scaring are also considered to influence axonal outgrowth from grafted neurons [16]. Park et al. found bioscaffolds highly effective to facilitate axonal growth from grafts after hypoxic injury, which was otherwise inhibited [17]. Technically, labeling strategies sufficient for the detection of distant processes were not always applied, which might have led to an underestimation of long-range projections in some studies. Mechanistically, axonal growth and pathfinding, as well as their inhibition, strongly depend on a precise interplay between endogenous signaling molecules and receptors on donor cells. Axonal outgrowth for example is, in the adult brain, inhibited by proteins associated with CNS myelin (e.g., Nogo) via signaling through respective receptors on growth cones [18]. However, why axonal outgrowth from some donor populations escapes the inhibitory environment in the adult brain while it is blocked for others has not yet been determined. Species differences might contribute to the different results obtained in various xenograft models, potentially due to a mismatch of endogenous inhibitory molecules with xenogeneic receptors on human neurons. However, this notion is challenged by data from Gaillard et al., showing that long-range axonal outgrowth is possible following transplantation of murine cells into adult isogenic hosts [19]. Moreover, the results of this and several other recent studies suggest that murine donor cells exhibiting the same regional identity as the implantation site can establish region-specific axonal projections in newborn [20, 21] and adult hosts [19]. However, the potential of human neural grafts to establish axonal projections in the adult brain still deserves further investigation.

We have recently established a stable population of long-term self-renewing neuroepithelial stem cells (lt-NES cells) from pluripotent human ES cells [2], which give rise to neurons with a posteriorized regional phenotype in vitro. We used this highly uniform population to explore whether heterotopically grafted human neural stem cells with a highly restricted regional phenotype can give rise to region-specific axonal projections. To that end, lt-NES cells were transplanted into the cortex and hippocampus of adult rodents, i.e., locations exhibiting different and highly specific neuronal innervation patterns of clinical relevance. Our data show that lt-NES-derived neurons develop axonal projections highly specific for the implantation site and establish morphologically mature synapses.

\section{Materials and methods}

Cell culture

Human ES cell-derived long-term self-renewing neuroepithelial stem cells (lt-NES cells, derived from hES cell lines
I3 and H9.2) were generated and transduced for GFP expression as described previously [2, 22] and in Supplementary Methods. For transplantation, donor cells passage 25-55 were trypsinized, washed in calcium- and magnesium-free PBS supplemented with $0.1 \%$ DNAse and concentrated to $7.5 \times 10^{4}$ cells $/ \mu$ l.

\section{PCR}

RNA was extracted using standard procedures from lt-NES cells and their differentiating progeny after 2, 4, and 8 weeks of in vitro differentiation and subsequently, cDNA was generated. Primer pairs (Supplementary Methods, table 1) were designed (Primer3) and PCRs performed using common cycling parameters.

Animals and transplantation

Severe combined immunodeficient-beige (SCID-bg) mice were used at an age of $8-10$ weeks $(n=52$, body weight 22-28 g). Alternatively, female 12-week-old SpragueDawley rats ( $n=20$, body weight $220-280 \mathrm{~g}$ ) were used. Rats and mice were stereotactically transplanted according to coordinates adopted from Paxinos et al. [23] (Supplementary Methods). Sprague-Dawley rats were immunosuppressed with daily injections of cyclosporine $(10 \mathrm{mg} / \mathrm{kg}$ i.p.). Animals were monitored for wound infections and neurological deficits on a daily basis during the first 2 weeks after transplantation and in weekly intervals thereafter. Care and use of the animals conformed to institutional policies and state legislation.

\section{Immunohistochemistry and microscopy}

Primary antibodies (Supplementary Methods, table 2) of the same species were never used together to avoid cross reactivity. Primary antibodies were visualized using corresponding FITC, Cy3 or Cy5 conjugated secondary antibodies. Sections were analyzed on a Fluoview 1000 confocal microscope (Olympus) or, if DAPI visualization was required, on a Zeiss Axioimager Z1 equipped with the Apotome technology (Zeiss) to reconstruct optical sections. Pre-embedding immunolabeling for electron microscopy was performed with the human specific anti-synaptophysin antibody. Ultrathin sections were examined under an electron microscope (CM-10, Philips).

Statistical analysis

In vivo analysis for the assessment of viability was performed in analogy to the Cavalieri method (Supplementary Methods). For determination of phenotypes in vivo at least 150 cells per animal ( $n=3$ per time point) were counted 
for every marker. Values represent $\% \pm$ standard deviation. Statistical significance was calculated using paired Student's $t$ test $[* p$ (two-sided) $=0.01-0.05]$.

\section{Results}

Prolonged differentiation into mature, non-tumorigenic grafts

Human ES cell-derived long-term self-renewing neuroepithelial stem cells (It-NES cells) were propagated as described previously [2]. In the presence of FGF2 and EGF, these cells exhibit uniform expression of the neural stem cell-associated genes nestin and sox 2 , a rosette-like growth pattern, high neurogenic differentiation potential, and a regional phenotype corresponding to an anterior hindbrain location, with all these properties remaining stable for at least 80 passages [2]. For transplantation, $7.5 \times 10^{4}$ lt-NES cells (passage 25-55; derived from lines I3 and H9.2 [24, 25]) expressing EGFP from the PGK promoter [2], were stereotactically injected into the dentate gyrus or motor cortex of adult immunodeficient SCID-bg mice or immunosuppressed Sprague-Dawley rats. Recipient mouse brains were analyzed 3, 6, 12, 24, and 48 weeks after transplantation, rat brains at 3,6 , and 12 weeks after transplantation.

Analysis of the hippocampal grafts in mice revealed that the survival of GFP-positive donor cells decreased from $48.7 \pm 5.5 \% 3$ weeks after transplantation to $15.8 \pm 4.8 \%$ 12 weeks after transplantation in the hippocampus and remained largely stable thereafter. In parallel, the graft volume decreased from $100.7 \pm 8.6 \times 10^{6}$ to $30.1 \pm$ $6.3 \times 10^{6} \mu \mathrm{m}^{3}$. Despite almost stable donor cell numbers beyond 12 weeks post grafting, the graft volume decreased further until the end of the experiment ( $p=0.03$, Fig. 1A). The majority of individual donor cells showed protracted differentiation characteristics along the neuronal lineage (Fig. 1B). Cells expressing nestin decreased from $39.2 \pm 10.0 \%$ at 3 weeks after transplantation to undetectable levels at $>6$ months after transplantation. Within the same time period, the percentage of proliferating Ki67positive cells decreased from $15.9 \pm 6.4 \%$ to undetectable levels (Fig. 1B-F). Not a single teratoma or neurogenic tumor was detected in more than 70 animals upon both macroscopic and microscopic examination. Immunohistochemical analysis of the grafts failed to detect any human cells positive for Oct 4 , cytokeratin, $\alpha$-fetoprotein and smooth muscle actin as markers for residual pluripotent or non-neural cells (data not shown). The neuronal marker MAP2ab (Fig. 1G) was present from the earliest time point of analysis and increased up to $82.7 \pm 3.3 \%$ at 12 months after implantation, whereas NeuN, another marker expressed in mature human neurons, was expressed only after more than 12 weeks in vivo and increased up to $24.7 \pm 9.3 \%$ at 12 months post grafting $(p=0.04$, Fig. 1H). Most of the grafted cells remained within the primary transplantation site (Fig. 1C, E, G). However, occasional mature neurons $(<0.1 \%)$ were found to populate adjacent hippocampal and cortical regions, where they could be detected for up to 60 weeks after transplantation, the latest time point assessed (Figs. 1H, 3C). GFAP-positive astrocytes accounted for less than $0.3 \%$ of detectable donor cells, and staining with the oligodendrocyte-specific marker $\mathrm{O} 4$ revealed no immunopositive cells (data not shown).

Donor cells could not only be identified by virtue of their GFP expression, but also using human-specific antibodies to human nuclei, Ki67, nestin, synaptophysin and NF-M [26]. Furthermore, human nuclei show a remarkably homogenous heterochromatin pattern in DAPI stains (Fig. 1D, H, asterisks), which can be distinguished from the coarse heterochromatin of mouse cells (e.g., Figs. 1H, 3H). Nonetheless, fusion of donor cells with host cells remains a concern in the interpretation of transplant studies, in particular when donor cells appear to acquire traits of resident cells. It is thus essential to distinguish whether regional differentiation patterns are truly due to donor cell plasticity rather than mimicry through cell fusion. We addressed this issue and stained grafted brain slices 6 and 9 months after transplantation into SCID-bg mice with an antibody against human nuclei and combined this staining with an in situ hybridization for the detection of mouse satellite DNA. More than 500 human nuclei were analyzed in both transplantation sites, but not a single nucleus co-stained positive for the murine DNA in situ probe (Fig. 1I), suggesting that cell fusion is not a relevant event after transplantation of human lt-NES cells into the adult mouse brain.

Human lt-NES cells acquire a posterior phenotype corresponding to the hindbrain area under standard in vitro differentiation conditions [2]. Therefore, we were interested whether these cells might, upon integration into the telencephalon, acquire an anterior phenotype. More than 150 donor cells were analyzed per animal and only two cells with a co-localization of human nuclei and BF1, a widely expressed telencephalic transcription marker, were found (Fig. 2A, <0.2\%). Mature human neurons were irrespective of their location of an inhibitory, GAD67positive (Fig. 2B, $55.8 \pm 6.8 \%$ ) and calretinin-positive phenotype (Fig. 2C) with no major difference in cortical versus hippocampal transplants. In addition, clusters of donor cells also stained positive for the excitatory synaptic marker vGlut2 (Fig. 2D). However, the punctate and distalsynaptic staining pattern of this marker precluded a reliable quantification. The remaining cells could not be clearly assigned. Specifically, no human nuclei or synapses 

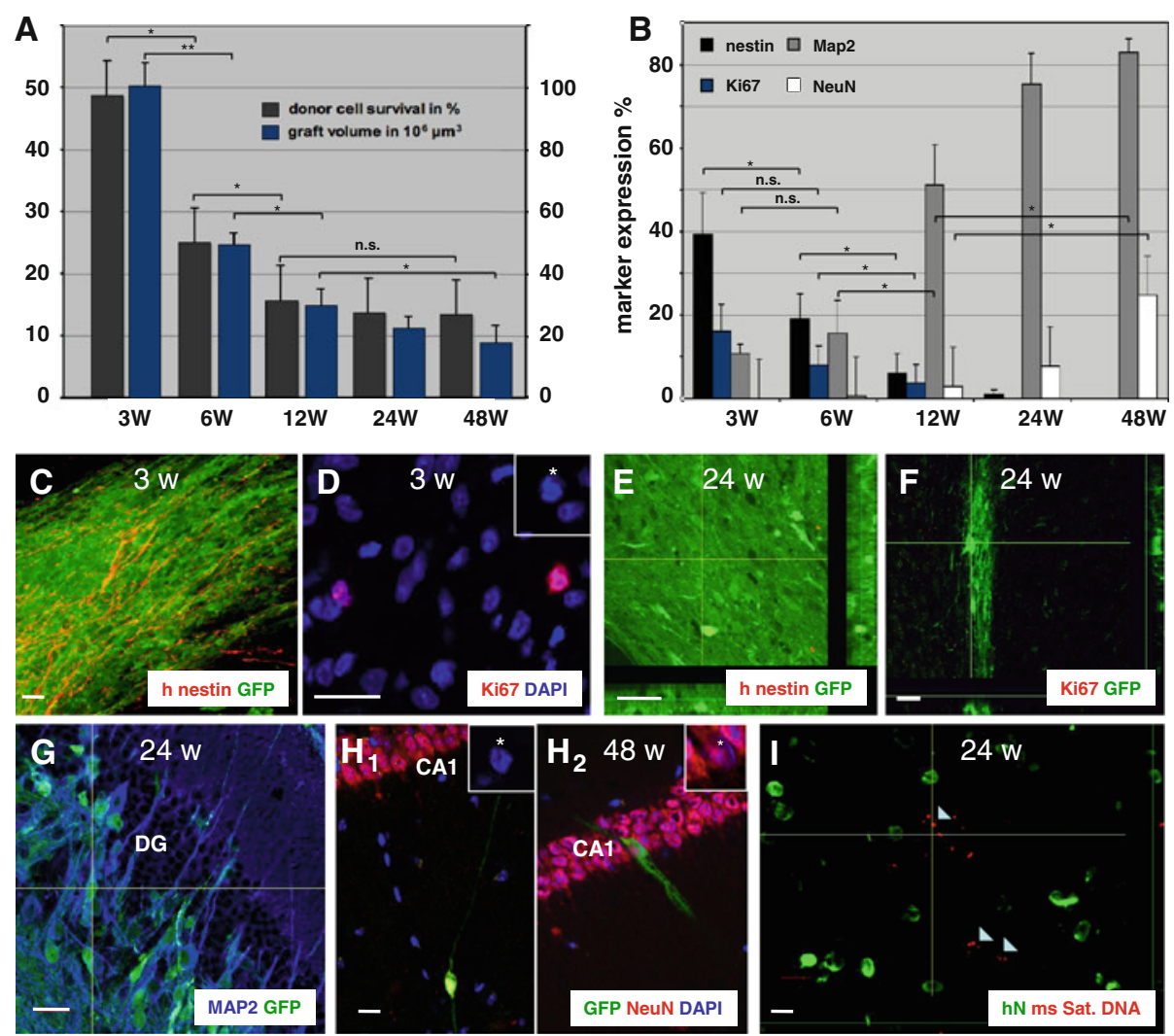

Fig. 1 Neurogenic differentiation of human lt-NES cells. A Analysis of donor cell survival and graft volume demonstrates increased cell death and shrinkage of the graft volume within the first 12 weeks (w) after hippocampal transplantation. B Quantification of marker expression in the grafted cells. Expression of nestin (C) and Ki67 (D) in a donor cell cluster within the motor cortex 3 weeks after transplantation. The insert in D depicts the DAPI staining of the Ki67 expressing cell showing a mitotic figure. Twenty-four weeks after transplantation expression of nestin $(\mathbf{E})$ and $\mathrm{Ki} 67(\mathbf{F})$ had decreased to undetectable levels. G Prominent neurogenic differentiation with detection of GFP- and MAP2-positive donor cells in a hippocampal

co-localized with vGlut1 or markers of dopaminergic or serotonergic differentiation. This transmitter phenotype is also in line with the in vitro regional code of the donor cell population and further supports the heterotopic identity of the graft with respect to the telencephalic transplantation sites. Human cells also stained positive for the GABA-A receptor (Fig. S1A) and the GluR1 subunit (Fig. S1B) of the AMPA receptor within hippocampal and motor cortex locations.

\section{lt-NES-derived neurons generate axons in vitro} and in vivo

After initiation of in vitro differentiation by growth factor withdrawal, lt-NES cells formed a complex axonal network within several weeks (Fig. S2A). RT-PCR analyses showed that differentiated lt-NES cells express a number of factors and receptors known to be involved in axon outgrowth and graft 24 weeks after transplantation. H Forty-eight weeks after transplantation surviving human neurons express NeuN. Human identity of the cells is confirmed by GFP expression as well as the homogenous chromatin pattern (insert, $\mathrm{H}_{1}$ ). A human NeuN-positive cell integrated into the pyramidal cell layer is shown in $\left(\mathrm{H}_{2}\right)$. I Cell fusion between host and donor cells was ruled out by a combination of anti-human Nuclei (hN) immunostaining and DNA in situ hybridization with a probe to mouse satellite DNA (arrowheads). Asterisks in $\mathbf{D}$ and $\mathbf{H}$ indicate the typical homogenous DAPI staining pattern of human nuclei, "h" in antibody denotation stands for human-specific, scale bars $20 \mu \mathrm{m}$

guidance [27] (Fig. S2B). Members of the netrin, ephrin, semaphorin and robo/slit families of guidance molecules and receptors were expressed in proliferating lt-NES cells, but some of them decreased during in vitro differentiation, suggesting the presence of a cell-autonomous time window for the direction of axonal outgrowth after transplantation. On the contrary, within the adult mammalian brain, axonal growth is limited to an absolute minimum [27]. Molecular stop signals like Nogo, Myelin-associated glycoprotein (MAG), Myelin-oligodendrocyte glycoprotein (MOG) and the repulsive guidance molecule (RGM-A) [27] play an important role. These inhibitors signal through a receptor complex composed of the Nogo receptor (NgR1), LINGO and p75. RT-PCR analysis of proliferating and differentiating human lt-NES cells revealed a continuous expression of Nogo and RGM-A, whereas MAG and MOG were not expressed (Fig. S2C). NgRs and Lingo were expressed at a constant level, whereas p75 was upregulated upon neuronal 

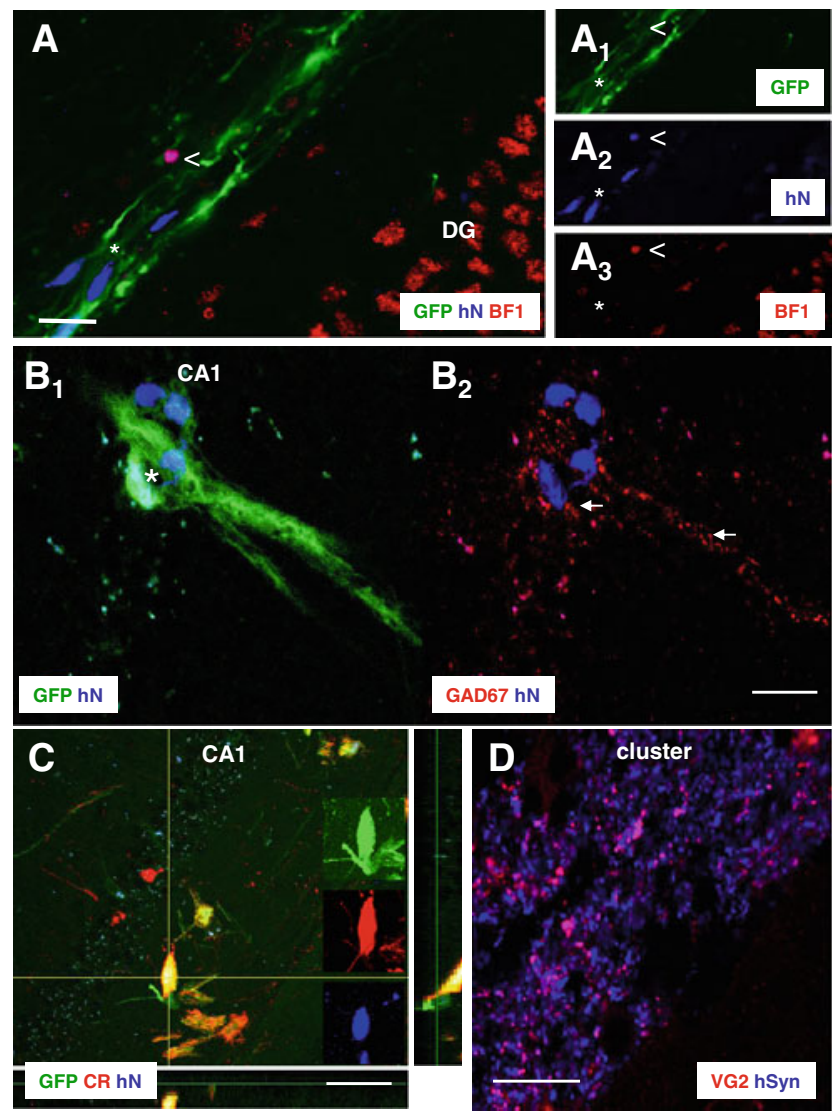

Fig. 2 Donor cells retain their phenotype in vivo. A Human donor cells rarely acquire markers of telencephalic identity (BF1) after transplantation. B A group of four human neurons identified by their GFP and human nuclei (hN)-positive staining within the CA1 pyramidal cell layer 9 months after transplantation of which the two lower cells show the mature punctuate expression pattern of the GABA producing enzyme GAD67 extending into the axonal processes. C A GFP/human nuclei double-positive cell within the stratum oriens of the CA1 sector showing strong calretinin (CR) expression. D The excitatory marker vGlut 2 was co-expressed with human synaptophysin positive punctae in clusters of donor cells. Scale bars $20 \mu \mathrm{m}$

maturation (also reflected at the immunohistochemical level in Fig. S3).

Upon in vivo transplantation, axons from primary motor cortex grafts grew with a speed of up to $1 \mathrm{~mm} /$ week within the first 6 weeks after transplantation in mice and rats as measured by the expression of the human neurofilament protein (hNF-M) (schematic representation in Fig. 3A, seen in 11 out of 12 mice surviving for 6 weeks or longer). Most cells remained within their primary cortical transplantation clusters (Fig. 3B) with single cells $(<0.1 \%$ ) migrating out of these primary transplantation sites (Fig. 3C). Human axons originating from these cells entered and followed the corpus callosum in the ipsilateral hemisphere and frequently branched off into the adjacent neocortex or entered the internal capsule (Fig. 3D). Upon high power magnification the grey matter of the basal ganglia, too, was scattered with axons staining for human synaptophysin. Patches of human synaptophysin immunoreactivity were frequently found in close spatial relationship with dots staining for the postsynaptic marker PSD95, suggesting the formation of xenogenic synapses (Fig. 3d, inset). Some of the donor-derived axons entered the cerebral peduncles (Fig. 3E) and could be further followed into the ipsi- and contralateral grey and white matter of the cervical spinal cord in 3 out of 8 mice surviving for 9 or 12 months after transplantation (Fig. 3F). Long-range projections were also found to extend through the corpus callosum into the contralateral hemisphere, where they branched off the corpus callosum and proceeded through all cortical layers (Fig. 3G). Here and in other target regions, human synaptophysin-positive dots were often closely associated with host axons, suggesting the formation of synaptic structures (Fig. 3H).

For hippocampal transplants, $7.5 \times 10^{4}$ cells were stereotaxically delivered to the upper blade of the dentate gyrus (DG) of adult mice and rats (Fig. 4B). Many axons emanating from these grafts projected to the ipsilateral CA3 sector, an innervation pattern characteristic of the mossy fiber pathway. Here, human axons, identified by virtue of their GFP expression, were found to spread across a distance of up to $2 \mathrm{~mm}$ within 3 weeks after transplantation (Fig. S4, rat hippocampus). Within the CA3 sector, human axons followed the stratum radiatum of the pyramidal layer (Fig. 4C), wherein they further projected to reach the adjacent CA2 and CA1 sectors. Remarkably, some of the donor-derived axons traversed the pyramidal cell layer, entered the fimbria and crossed to the contralateral hemisphere, where they approached the contra-lateral hippocampal pyramidal cell layer (Fig. 4D), a trajectory typical for commissural hippocampal axons. Quantification of axons positive for human neurofilament revealed a high abundance of donor-derived fibers in the ipsi- and contra-lateral hippocampus as compared to extrahippocampal regions such as the corpus callosum $(p=0.004)$, the entorhinal $(p=0.005)$ or motor cortex and the thalamus (Fig. 4E). This data strongly supports the notion that the engrafted neurons adopt projection patterns typical for resident hippocampal neurons [28].

\section{Evidence for the formation of xenogenic synapses}

Considering the well-characterized synaptic circuitry of the hippocampus, we chose hippocampal grafts to assess morphologically the formation of synapses between donor and host cells. At 6 months after transplantation, human axons identified with the human-specific NF-M antibody were found inside the stratum radiatum (STR; Fig. 5A). Alongside these projections, small patches of human synaptophysin immunoreactivity were detected, suggesting the 

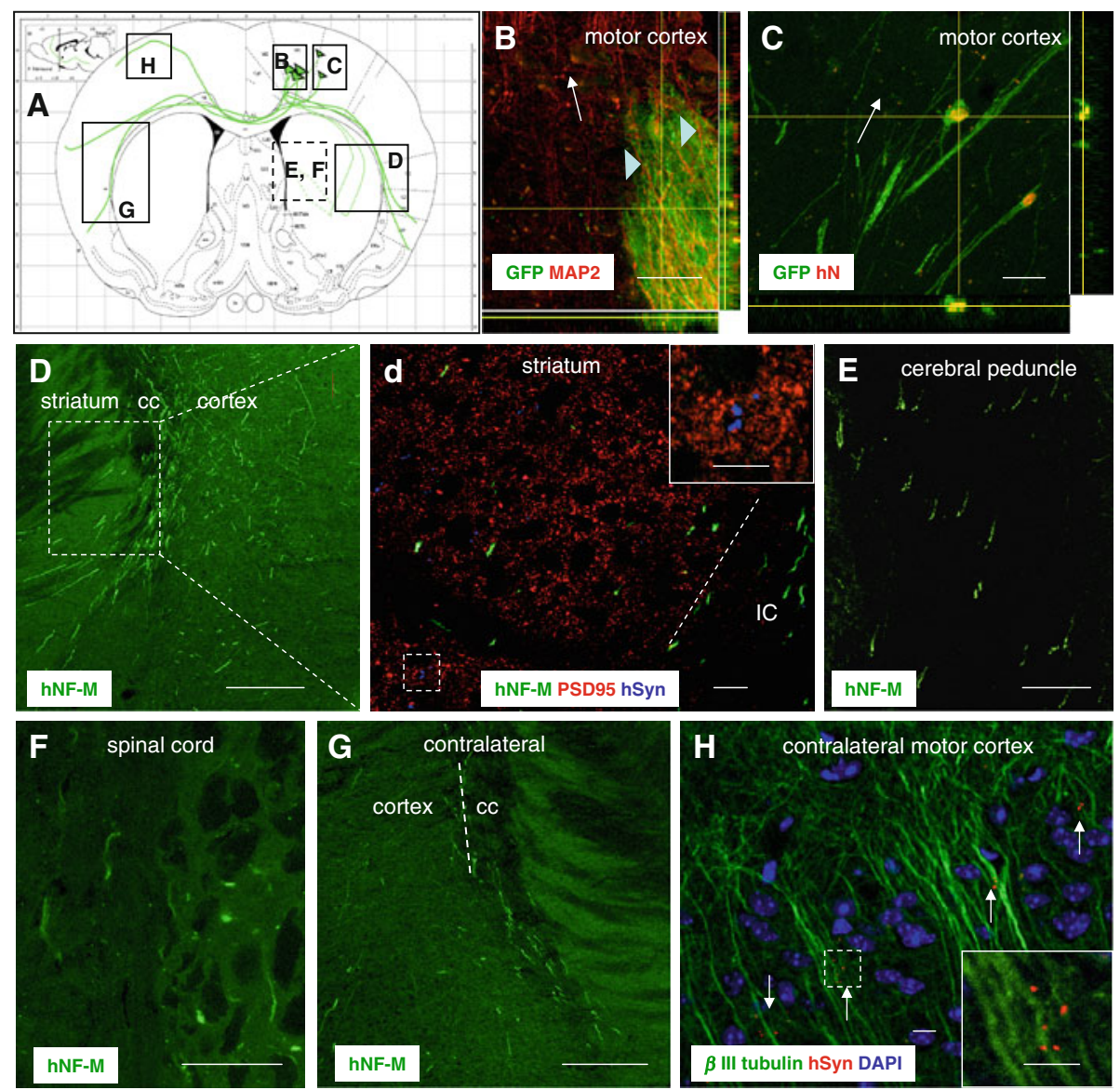

Fig. 3 Axon outgrowth from motor cortex transplants. A schematic representation of the transplant site within the primary motor cortex and corresponding axonal outgrowth is depicted in A. A GFP-positive microtransplant within the lower layers of the motor cortex 6 months after transplantation is shown in B with GFP/MAP2-positive dendrites (arrowheads) extending into superficial cortical layers. Single human neurons migrated out of the clusters for a maximum distance of $1.5 \mathrm{~mm}$, still residing within the cortex where they oriented radially $\mathbf{C}$. Most human fibers entered and proceeded within the corpus callosum (CC), frequently branched off into the adjacent cortex, or turned medial to join the internal capsule (IC) D. Magnification from the striatum $\mathbf{d}$ revealed human fibers within the white

formation of presynaptic terminals. Some of these patches were co-stained with an antibody to vGlut2 (dilution 1:4,000, boxed areas in Fig. 5B). No vGlut2 immunoreactivity was detected outside the hSyn-positive patches under the conditions used here. No hSyn or vGlut 2 signal was detected in non-transplanted hippocampi. When the vGlut2 antibody was used at higher concentrations (1:800), abundant small immunoreactive puncta became detectable (data not shown), most likely corresponding to the endogenous vGlut2 positive terminals in this region [29]. vGlut2 may thus, under the special conditions described here, be considered as a human-specific marker. In further triple-labelings, the antibody against hSyn was replaced by a MAP2ab antibody labeling host dendrites (Fig. 5C). High matter of the internal capsule, and human-specific synaptophysin immunoreactivity, frequently in close association with PSD95 immunoreactivity (magnified insert). Human axons were also identified in the cerebral peduncle $\mathbf{E}$ and within the grey and white matter of the cervical spinal cord $\mathbf{F}$. Human axons crossed to the contralateral hemisphere branched off the corpus callosum and traversed through the adjacent cortex in a tangential orientation G. Here human-specific synaptophysin immunoreactivity was found in close association with axons of host neurons (magnified insert in $\mathbf{H})$. Scale bars $\mathbf{B}, \mathbf{C}, \mathbf{E}$ and $\mathbf{F} 30 \mu \mathrm{m}, \mathbf{D}$ and $\mathbf{G} 100 \mu \mathrm{m}, \mathrm{d}$ and $\mathrm{H}$ $10 \mu \mathrm{m}$. In $\mathbf{B}$ and $\mathbf{C}$ arrows point to pial surface

magnification revealed a dotted vGlut2-positive staining of human synaptic terminals around host dendrites, suggesting the formation of xenogenic synapses (Fig. 5c). Interestingly, in the ipsilateral hippocampus hSyn and vGlut2 immunoreactivity were mainly detected within the stratum radiatum (STR, $p=0.015$ ), whereas within the contralateral hippocampus (Fig. 5D) vGlut2 immunoreactivity was, though generally less abundant (STR ipsilateral vs. STR contralateral $p=0.049$ ), preferentially found in the vicinity of human fibers within the stratum oriens (Fig. 5E).

The clear identification of hSyn-positive terminals and co-localization with markers for specific neurotransmitters allowed the quantification of inhibitory and excitatory 

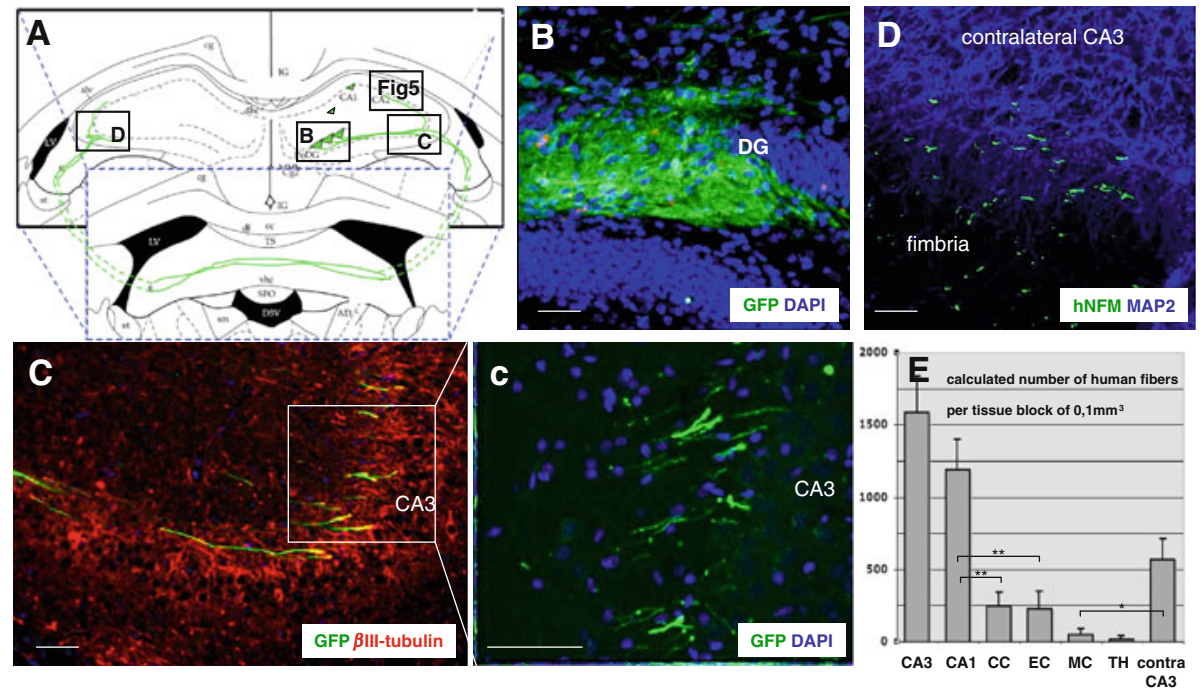

Fig. 4 Axon outgrowth from hippocampal transplants. A Schematic representation of the transplant site within the dentate gyrus (DG) and corresponding axonal outgrowth. B GFP-positive microtransplant within the DG of an adult mouse 3 months after transplantation. While axonal outgrowth from these transplants preferentially approached the ipsilateral CA3 region ( $\mathbf{C}$, high magnification detail in $\mathbf{c})$, a significant number of donor fibers also reached the contralateral CA3 sector (D).

donor-derived synaptic terminals in different projection fields. This analysis revealed that within the ipsilateral pyramidal cell layer, $36.7 \pm 5.9 \%$ of human terminals costained positive for GAD67 whereas $12.8 \pm 3.2 \%$ of the human terminals co-stained positive for vGlut 24 weeks after transplantation. Within the contralateral pyramidal cell layer $32.9 \pm 5.9 \%$ of the human terminals stained positive for vGlut2 and no clear co-localization with GAD67 could be detected.

To confirm the formation of xenogenic synapses at the ultrastructural level we performed preembedding electron microscopy with an antibody recognizing human synaptophysin 1 year after transplantation. In line with the light microscopic data, strong immunoreactivity was selectively found in axon terminals (Fig. 6), demonstrating correct protein targeting of synaptophysin in the human lt-NES cell-derived neurons. Stained terminals formed regular synaptic contacts in the hilus close to the transplant (Fig. 6A) and within the ipsilateral stratum radiatum of $\mathrm{CA}$ 1-3 (Fig. 6B, C). As a structural sign of functional activity, the terminals displayed abundant vesicles, and in particular docked vesicles at the presynaptic membrane (Fig. 6a-c), suggesting the presence of a readily releasable pool of vesicles [30].

\section{Discussion}

The most important finding of this study is the remarkable specificity with which human ES cell-derived long-term
E Quantification of human fibers in tissue blocks of a defined volume revealed a mean density of $1,125-1,575$ fibers $/ 0.1 \mathrm{~mm}^{3}$ within the ipsilateral CA3-CA1 sector and 675 fibers $/ 0.1 \mathrm{~mm}^{3}$ within the contralateral CA3 sector. In contrast, projections to extra-hippocampal regions such as corpus callosum (CC), entorhinal cortex (EC), motor cortex (MC) and thalamus (TH) were sparse (all <270 fibers/ $\left.0.1 \mathrm{~mm}^{3}\right)$. Scale bars $50 \mu \mathrm{m}$

self-renewing neuroepithelial stem cells (lt-NES) recapitulate endogenous axonal projections within the adult brain. Some previous studies with primary and in vitro propagated human cells had already hinted at their capacity for extensive axonal innervation $[9,10,15,31]$, which was mainly found to follow white matter tracts close to the site of transplantation. Our study revealed that upon transplantation into the motor cortex lt-NES establish ipsi- and contralateral projections as well as trajectories into the pyramidal and extrapyramidal motor system, including the cerebral peduncles and the cervical spinal cord. Interestingly, the same cells adopted a hippocampus-specific projection profile with laminar specificity when transplanted into the dentate gyrus. The contralateral fiber projection and termination pattern via the fimbria-fornix closely resembles that of endogenous commissural fibers [28]. Importantly, only glutamatergic human terminals were detectable along these long-range fibers, despite the fact that the majority of donor cells show a GABAergic phenotype after transplantation. To exclude fusion with host cells as a possible explanation for this phenomenon, we used all available genetic, immunological, and morphological markers to unambiguously identify human cells. We also demonstrate that human axon terminals establish contacts displaying all morphological characteristics of normal active synapses.

So far, only few studies employing murine cells have reported such a highly region-specific projection pattern of grafted neurons. Gaillard et al. [19] showed that motor cortex grafts can reestablish appropriate long-range 
Fig. 5 Immunohistochemistry of xenogenic synapses. A Axons positive for human neurofilament (hNF-M) project through the stratum radiatum of CA1, which exhibits abundant dotty immunoreactivity for human synaptophysin, suggesting formation of presynaptic terminals by the donor neurons. B Triple immunofluorescence staining reveals that a subset of human synapses co-expresses the glutamatergic marker vGlut2 (boxed purple punctae). C Triple staining with antibodies to hNF-M, vGlut2 and MAP2 reveals a close association of the human glutamatergic terminals with host dendrites (c shows magnification of the boxed area in $\mathbf{C})$. $\mathbf{D}$ in the contralateral CA3 sector vGlut2 immunoreactivity localizes mainly within the stratum oriens and the pyramidal cell layer, indicated by arrowheads, as quantified in E. PCL pyramidal cell layer, STR stratum radiatum, STO stratum oriens, scale bars $20 \mu \mathrm{m}$
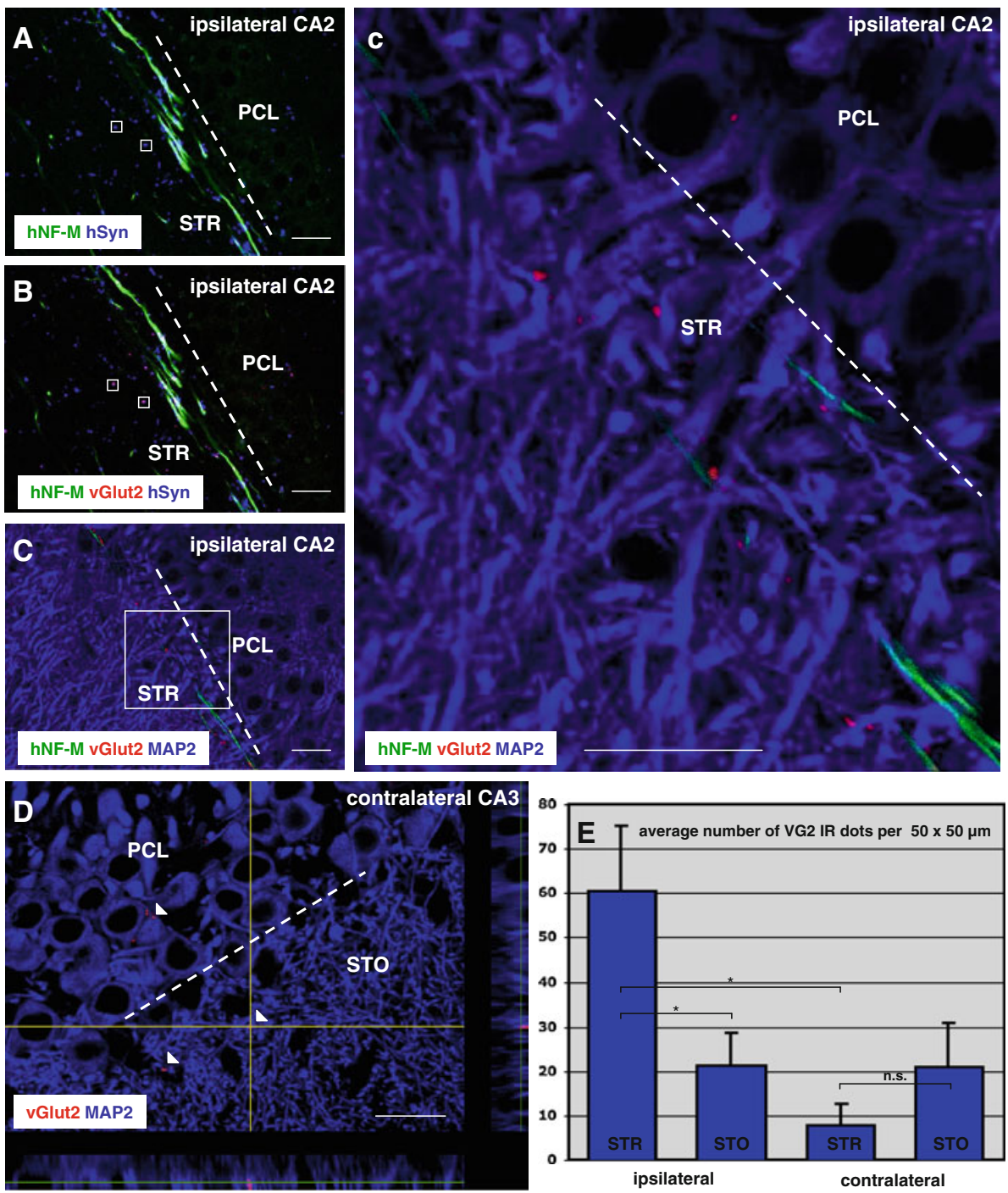

projections within the adult murine motor system. They found the homotopic nature of their explants to be important for successful reconstruction. This notion is further supported by two recent publications employing transplantation of murine ES cell-derived cortical precursors into newborn mice [20, 21]. In contrast, the human ES cellderived 1t-NES cells used in our study do not exhibit a telencephalic phenotype. They are posteriorized and show a marker profile compatible with an anterior hindbrain identity, a bias acquired during long-term in vitro expansion in the presence of growth factors [2]. After transplantation into the adult hippocampus and motor cortex, they do not acquire a region-specific phenotype as indicated by the absence of BF1, a transcription factor broadly expressed in both telencephalic regions. Thus, for both target regions, our donor cell population can be regarded as heterotopic. Yet, the cells exhibit highly specific patterns of axonal outgrowth.
The precise mechanisms for this remarkable specificity remain to be elucidated. As in the study by Gaspard et al., we left the surrounding endogenous motor cortex or hippocampal tissue intact in order to maintain potential local guidance cues [27]. However, it seems unlikely that complex non-linear trajectories such as innervation of target regions in the contralateral hemisphere via the fimbriafornix pathway are merely guided by chemoattractants and repellents. A more likely explanation could be that the newly formed axons grow alongside host fiber tracts, which, by nature, represent region-specific trajectories. Considering that many of the newly formed axonal projections pass myelinated fiber tracts such as the corpus callosum and the fimbria-fornix, it is remarkable that this process appears not to be inhibited by myelin-associated inhibitors of axonal growth. In this respect, the slow maturation of human neurons might provide a substantial advantage. We found that p75 is only upregulated after 


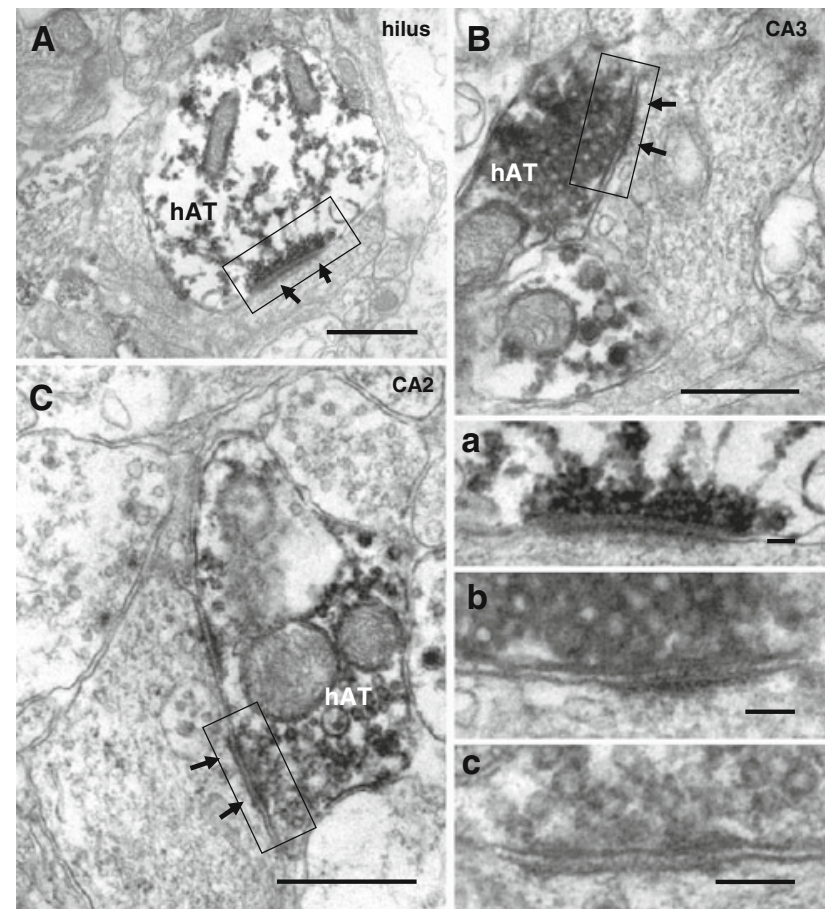

Fig. 6 Xenogenic axon terminals establish synapses on host cells. A-C Axon terminals positive for human synaptophysin display a black DAB reaction product. Despite the DAB precipitate, standard morphology of synaptic contacts (bold arrows in $\mathbf{A}-\mathbf{C}$ ) can be clearly discerned. a-c Magnified synapses (from boxed areas in A-C) show further details, such as presynaptic vesicle clusters, docked vesicles at the presynaptic membrane, pre- and postsynaptic densities and cleft material. As most transplant synapses, the examples shown in $\mathbf{A}-\mathbf{C}$ are asymmetric. $h A T$, human axon terminal, scale bars $1 \mu \mathrm{m} \mathbf{A}-\mathbf{C}, 0.2 \mu \mathrm{m}$ a-c

several weeks of in vitro differentiation. This delayed expression of an important member of the receptor complex inhibiting axonal outgrowth $[32,33]$ might determine a permissive time window for axonal outgrowth from human lt-NES cells in the adult brain.

\section{Prospects for experimental brain repair}

Although the mechanisms enabling long-range axonal projections in the adult mammalian nervous system require further investigation, the results of our and other studies in related systems $[9,10,15,31]$ indicate that human neural grafts may, under appropriate conditions, eventually be used for the innervation of remote targets in the host brain. This prospect could be particularly relevant for diseases affecting a specific group of neurons with defined projections. Examples include central motoneurons, affected in ALS, but also nigral dopamine neurons projecting into the striatum, the main target of Parkinson's disease. In this regard, it is important to realize that substantial functional benefits might already result from incomplete structural repair [34]. However, functional data going beyond the morphological detection of axons and synapses will be essential to validate the efficacy of graft-derived projections.

Safety considerations play an essential role with respect to the source of the donor cells $[35,36]$. The potential to proliferate human ES cell-derived lt-NES cells over many passages without compromising their neuronal differentiation potential enables the generation of large numbers of pure neural donor cells. In our preclinical model, and with the cell numbers used, this population did not result in tumor/teratoma formation in any of the transplant recipients, which were followed up to the limit of the recipient animal's lifespan.

Taken together, human neuroepithelial stem cells derived from pluripotent cells show promising results in terms of transplant survival, safety, neuronal differentiation, axonal pathfinding, and synaptogenesis in vivo. In combination with efficient strategies to direct the donor cells towards fates of therapeutic value [37-41] and iPS technology $[36,42]$ for the generation of immunocompatible grafts, they should provide a versatile tool for experimental nervous system repair.

Acknowledgments We are grateful to Joseph Itskovitz-Eldor (Technion, Israel Institute of Technology, Haifa, Israel) for providing the hESC lines I3 and H9.2. We thank Michaela Segschneider, Anke Leinhaas, Christina Leufgen, and Justus Ritter for outstanding technical support. We would further like to thank Jörg Bedorf and Reinhard Büttner for their support regarding the EM studies. This work was supported by Deutsche Forschungsgemeinschaft Grants SFB-TR3 (TP D2 and TP C1), European Union Grant FP7-HEALTH2007-B-22943-NeuroStemcell and the Hertie Foundation.

Open Access This article is distributed under the terms of the Creative Commons Attribution Noncommercial License which permits any noncommercial use, distribution, and reproduction in any medium, provided the original author(s) and source are credited.

\section{References}

1. Elkabetz Y, Panagiotakos G, Al Shamy G et al (2008) Human ES cell-derived neural rosettes reveal a functionally distinct early neural stem cell stage. Genes Dev 22:152-165

2. Koch P, Opitz T, Steinbeck JA et al (2009) A rosette-type, selfrenewing human ES cell-derived neural stem cell with potential for in vitro instruction and synaptic integration. Proc Natl Acad Sci USA 106:3225-3230

3. Zhang SC, Wernig M, Duncan ID et al (2001) In vitro differentiation of transplantable neural precursors from human embryonic stem cells. Nat Biotechnol 19:1129-1133

4. Kallur T, Darsalia V, Lindvall O et al (2006) Human fetal cortical and striatal neural stem cells generate region-specific neurons in vitro and differentiate extensively to neurons after intrastriatal transplantation in neonatal rats. J Neurosci Res 84:1630-1644

5. Sabate O, Horellou P, Vigne E et al (1995) Transplantation to the rat brain of human neural progenitors that were genetically modified using adenoviruses. Nat Genet 9:256-260 
6. Uchida N, Buck DW, He D (2000) Direct isolation of human central nervous system stem cells. Proc Natl Acad Sci USA 97:14720-14725

7. Walton NM, Sutter BM, Chen HX et al (2006) Derivation and large-scale expansion of multipotent astroglial neural progenitors from adult human brain. Development 133:3671-3681

8. Koch P, Kokaia Z, Lindvall O et al (2009) Emerging concepts in neural stem cell research: autologous repair and cell-based disease modelling. Lancet Neurol 8:819-829

9. Englund U, Bjorklund A, Wictorin K (2002) Migration patterns and phenotypic differentiation of long-term expanded human neural progenitor cells after transplantation into the adult rat brain. Brain Res Dev Brain Res 134:123-141

10. Wictorin K, Brundin P, Gustavii B et al (1990) Reformation of long axon pathways in adult rat central nervous system by human forebrain neuroblasts. Nature 347:556-558

11. Fricker RA, Carpenter MK, Winkler C et al (1999) Site-specific migration and neuronal differentiation of human neural progenitor cells after transplantation in the adult rat brain. J Neurosci 19:5990-6005

12. Svendsen CN, Clarke DJ, Rosser AE et al (1996) Survival and differentiation of rat and human epidermal growth factorresponsive precursor cells following grafting into the lesioned adult central nervous system. Exp Neurol 137:376-388

13. Vescovi AL, Parati EA, Gritti A et al (1999) Isolation and cloning of multipotential stem cells from the embryonic human CNS and establishment of transplantable human neural stem cell lines by epigenetic stimulation. Exp Neurol 156:71-83

14. Kelly CM, Precious SV, Penketh R et al (2007) Striatal graft projections are influenced by donor cell type and not the immunogenic background. Brain 130:1317-1329

15. Ostenfeld T, Caldwell MA, Prowse KR et al (2000) Human neural precursor cells express low levels of telomerase in vitro and show diminishing cell proliferation with extensive axonal outgrowth following transplantation. Exp Neurol 164:215-226

16. Bentlage C, Nikkhah G, Cunningham MG et al (1999) Reformation of the nigrostriatal pathway by fetal dopaminergic micrografts into the substantia nigra is critically dependent on the age of the host. Exp Neurol 159:177-190

17. Park KI, Teng YD, Snyder EY (2002) The injured brain interacts reciprocally with neural stem cells supported by scaffolds to reconstitute lost tissue. Nat Biotechnol 20:1111-1117

18. Schwab ME (2004) Nogo and axon regeneration. Curr Opin Neurobiol 14:118-124

19. Gaillard A, Prestoz L, Dumartin B et al (2007) Reestablishment of damaged adult motor pathways by grafted embryonic cortical neurons. Nat Neurosci 10:1294-1299

20. Gaspard N, Bouschet T, Hourez R et al (2008) An intrinsic mechanism of corticogenesis from embryonic stem cells. Nature 455:351-357

21. Ideguchi M, Palmer TD, Recht LD et al (2010) Murine embryonic stem cell-derived pyramidal neurons integrate into the cerebral cortex and appropriately project axons to subcortical targets. J Neurosci. 30:894-904

22. Koch P, Siemen H, Biegler A et al (2006) Transduction of human embryonic stem cells by ecotropic retroviral vectors. Nucleic Acids Res 34:e120

23. Paxinos G, Watson C (1997) The rat brain in stereotaxic coordinates. Academic Press, San Diego

24. Amit M, Carpenter MK, Inokuma MS et al (2000) Clonally derived human embryonic stem cell lines maintain pluripotency and proliferative potential for prolonged periods of culture. Dev Biol 227:271-278
25. Thomson JA, Itskovitz-Eldor J, Shapiro SS et al (1998) Embryonic stem cell lines derived from human blastocysts. Science 282:1145-1147

26. Trojanowski JQ, Mantione JR, Lee JH et al (1993) Neurons derived from a human teratocarcinoma cell line establish molecular and structural polarity following transplantation into the rodent brain. Exp Neurol 122:283-294

27. Harel NY, Strittmatter SM (2006) Can regenerating axons recapitulate developmental guidance during recovery from spinal cord injury? Nat Rev Neurosci 7:603-616

28. Frotscher M, Kugler P, Misgeld U et al (1988) Neurotransmission in the hippocampus. Adv Anat Embryol Cell Biol 111:1-103

29. Wallen-Mackenzie A, Nordenankar K, Fejgin K et al (2009) Restricted cortical and amygdaloid removal of vesicular glutamate transporter 2 in preadolescent mice impacts dopaminergic activity and neuronal circuitry of higher brain function. J Neurosci 29:2238-2251

30. Schneggenburger R, Sakaba T, Neher E (2002) Vesicle pools and short-term synaptic depression: lessons from a large synapse. Trends Neurosci 25:206-212

31. Nasonkin I, Mahairaki V, Xu L, et al. (2009) Long-term, stable differentiation of human embryonic stem cell-derived neural precursors grafted into the adult mammalian neostriatum. Stem Cells 27(10):2414-2426

32. Ahmed Z, Dent RG, Suggate EL et al (2005) Disinhibition of neurotrophin-induced dorsal root ganglion cell neurite outgrowth on CNS myelin by siRNA-mediated knockdown of $\mathrm{NgR}$, p75NTR and Rho-A. Mol Cell Neurosci 28:509-523

33. Ahmed Z, Mazibrada G, Seabright RJ et al (2006) TACE-induced cleavage of $\mathrm{NgR}$ and $\mathrm{p} 75 \mathrm{NTR}$ in dorsal root ganglion cultures disinhibits outgrowth and promotes branching of neurites in the presence of inhibitory CNS myelin. FASEB J. 20:1939-1941

34. Freund P, Schmidlin E, Wannier T et al (2006) Nogo-A-specific antibody treatment enhances sprouting and functional recovery after cervical lesion in adult primates. Nat Med 12:790-792

35. Roy NS, Cleren C, Singh SK et al (2006) Functional engraftment of human ES cell-derived dopaminergic neurons enriched by coculture with telomerase-immortalized midbrain astrocytes. Nat Med 12:1259-1268

36. Wernig M, Zhao JP, Pruszak J et al (2008) Neurons derived from reprogrammed fibroblasts functionally integrate into the fetal brain and improve symptoms of rats with Parkinson's disease. Proc Natl Acad Sci USA 105:5856-5861

37. Andersson E, Tryggvason U, Deng Q et al (2006) Identification of intrinsic determinants of midbrain dopamine neurons. Cell 124:393-405

38. Eiraku M, Watanabe K, Matsuo-Takasaki M et al (2008) Selforganized formation of polarized cortical tissues from ESCs and its active manipulation by extrinsic signals. Cell Stem Cell 3:519-532

39. Lee G, Kim H, Elkabetz Y et al (2007) Isolation and directed differentiation of neural crest stem cells derived from human embryonic stem cells. Nat Biotechnol 25:1468-1475

40. Lee H, Shamy GA, Elkabetz Y et al (2007) Directed differentiation and transplantation of human embryonic stem cell-derived motoneurons. Stem Cells. 25:1931-1939

41. Li T, Steinbeck JA, Lusardi T et al (2007) Suppression of kindling epileptogenesis by adenosine releasing stem cell-derived brain implants. Brain 130:1276-1288

42. Takahashi K, Tanabe K, Ohnuki M et al (2007) Induction of pluripotent stem cells from adult human fibroblasts by defined factors. Cell 131:861-872 